\title{
Novel Variable Coupling Technique for Microwave Liquid Heating and Sensing
}

\author{
Ali A. Abduljabar, Heungjae Choi, Member, IEEE, and Adrian Porch
}

\begin{abstract}
A simple, new, variable-coupling technique is presented in this paper. The aim of the design is to optimize the coupling between a microwave source and a microwave resonator in which the resonator (in our case a cylindrical cavity resonator, for demonstration purposes) can be used either as a microwave applicator or sensor. Our technique involves the inclusion of a variable capacitor in the coupling loop that terminates the cavity's coaxial feedline. . For proof-of-concept, the proposed approach was applied to a $2.45 \mathrm{GHz} \mathrm{TM}_{010}$ cylindrical cavity for heating and sensing of several types of solvents. The experimental results show the capability to reach any coupling value when the resonator is loaded with various liquid samples by adjusting the value of the terminating capacitor. Hence, the critical coupling condition can be achieved by electronic, rather than mechanical, means.
\end{abstract}

Index Terms-Microwave applicator, heating, resonator, sensor.

\section{INTRODUCTION}

$\mathrm{O}$ $\mathrm{NE}$ of the key requirements in medical, biological, and chemical applications is the combination of the microwave heating and sensing techniques, where the same microwave resonator is used for heating and characterization of the sample at the same time. In [1] and [2], a planar structure based on a split ring resonator was used to design dual sensor which can be operated for sensing the dielectric properties of the organic tissue as well as for executing thermal ablation therapy. The simultaneous sensing and heating of individual nanoliter-sized droplets in microfluidic devices was presented in [3], where an electrically small resonator was able to distinguish between materials with different electrical properties. A $\mathrm{TE}_{011}$ cylindrical cavity was designed at $2.45 \mathrm{GHz}$ in [4] to heat and measure the temperature dependence for thin materials. A microwave cavity and heating system for microwave processing and in situ dynamic measurements of the complex permittivity of dielectric materials at high temperatures $\left(1000{ }^{\circ} \mathrm{C}\right)$ has been developed in [5], where a dual-mode cylindrical cavity was used with two different swept frequency microwave sources for heating and testing.

Ali A. Abduljabar is with the Department of Electrical Engineering, College of Engineering, University of Basrah, 61001 Basrah, Iraq (e-mail: aliaiq76@gmail.com).

H. Choi and A. Porch are with the School of Engineering, Cardiff University, 1-5 The Parade, Cardiff, CF24 3AA, United Kingdom (e-mail: choih1@cardiff.ac.uk; porcha@cardiff.ac.uk).

Color versions of one or more of the figures in this paper are available online via http://ieeexplore.ieee.org.

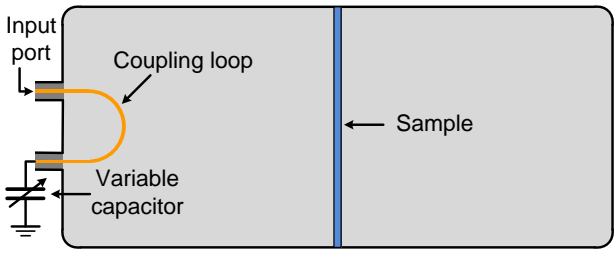

Fig. 1. Cross section of the cylindrical waveguide cavity with one input variable coupling circuit,

Another requirement in microwave heating applications is the improvement of the heating efficiency as the coupling varies for different samples (i.e. electrical loads). Also the coupling varies with temperature because the sample's permittivity is often highly dependent on temperature, as is the case with common polar solvents. Improved electromagnetic heating of a load was demonstrated by increasing the isolation (or decoupling) factor between two electromagnetic feed elements [6]. An electronically adaptive coupling technique is presented in [7] for a microwave microstrip resonator to improve the efficiency of liquid heating in a microfluidic system, where the resonator is fed with two synchronized inputs with a variable phase shifter between them.

In this paper, we propose a new coupling structure for a resonator used for simultaneous heating and sensing applications by using a variable coupling circuit, as shown schematically in Fig. 1. Moreover, the variable coupling circuit can be adjusted to obtain maximum coupling during the heating when the load (sample) changes its dielectric property with temperature. This approach was applied to $\mathrm{TM}_{010}$ mode in a cylindrical cavity at $2.45 \mathrm{GHz}$ loaded with several liquids to verify the variable coupling topology.

\section{DESIGN OF VARIABLE COUPLING STRUCTURE}

To achieve our goal, the coupling circuit must have the ability to reach critical coupling with the resonator working as an applicator so that maximum power can be delivered to the sample. Additionally, the coupling circuit should have the ability to reduce the coupling strength in the case when the resonator is switched into its sensing mode (i.e. for complex permittivity characterization) to increase the loaded quality factor to improve the accuracy of the measurement.

The voltage reflection coefficient at the input of the coupling circuit with one-port resonator can be expressed as: 


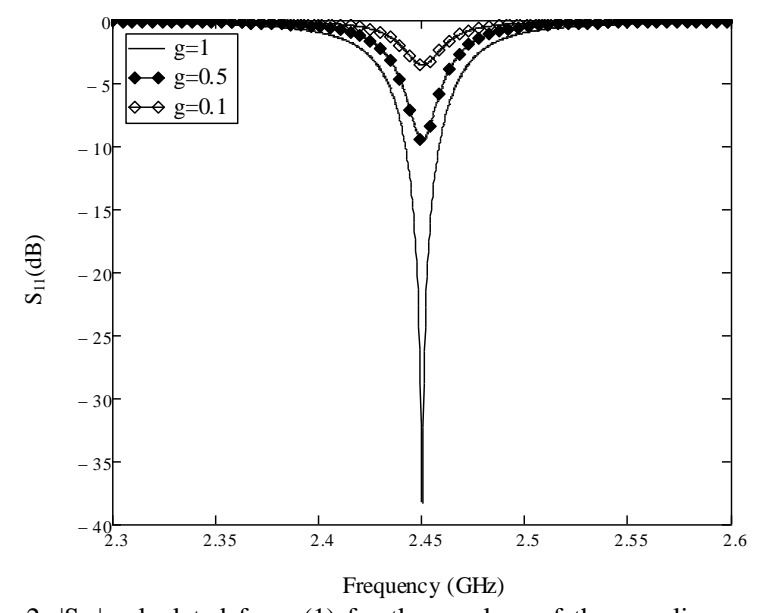

Fig. 2. $\left|S_{11}\right|$ calculated from (1) for three values of the coupling coefficient when the loaded quality factor is equal to 100 .

$$
S_{11} \approx \frac{g-1+2 j Q_{o}\left(\frac{\omega-\omega_{o}}{\omega_{o}}\right)}{g+1+2 j Q_{o}\left(\frac{\omega-\omega_{o}}{\omega_{o}}\right)}
$$

where $g$ is the coupling coefficient, defined as:

$$
g=\frac{\omega_{o}^{2} m^{2}}{Z_{o} R} \propto m^{2} Q_{0}
$$

where $\omega_{o}$ is the resonant frequency, $Q_{o}$ is the unloaded quality factor of the resonator, $m$ is the mutual inductance between the coupling loop and the resonator, $R$ is the resistance of the resonator (assuming a series $R L C$ equivalent circuit for the resonator), and $Z_{o}$ is the characteristic impedance of the input/output transmission lines (here $50 \Omega$ ). According to (1), if we assume that the unloaded quality factor of the cavity with a water sample is 100 and the resonant frequency is equal to $2.45 \mathrm{GHz}$, then the change in $\left|S_{11}\right|$ with respect to the coupling coefficient $g$ can be plotted as shown in Fig. 2. For $g$ in (2) to be variable, $m$ needs to change. The radius of the coupling loop cannot be modified once it is built, but the electrical length of the coupling loop can be changed by terminating the loop with a variable capacitor. This can also offset changes in $g$ due to changes in $Q_{o}$, thus ensuring the condition for maximum power transfer (i.e. $g=1$, critical coupling) can be maintained. As a proof of concept, a cylindrical cavity with a resonant frequency of $2.45 \mathrm{GHz}$ for $\mathrm{TM}_{010}$ mode is used. The electric field at the center of the cavity is parallel and equal to the internal electric field of the sample [8], therefore, the depolarization is a minimum and the maximum delivery of power to the sample will occur.

Fig. 3 shows the equivalent circuit of variable coupling structure shown in Fig. 1. The variable admittance is modeled as in [9] by means of the coupling variable element made up of the loop segment and the transmission line terminated with variable capacitor as:

$$
Y=Y_{v c}+\frac{Y_{c}}{j \tan \left(\theta_{c}\right)}
$$

where $Y_{v c}$ is the input admittance of the transmission line terminated with variable capacitor. $Y_{c}$ and $\theta_{c}$ are the

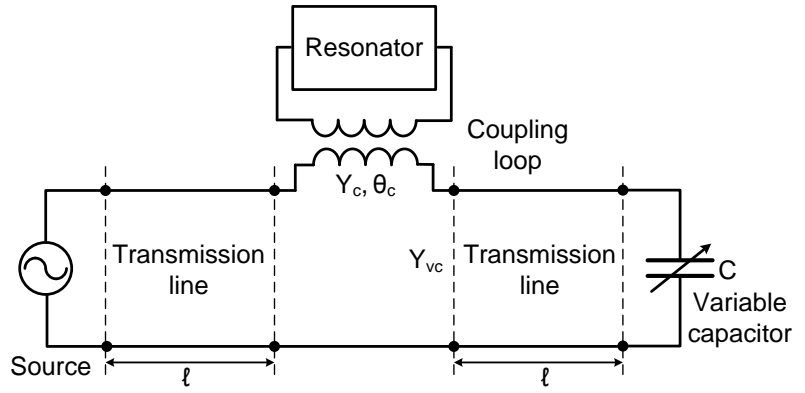

Fig. 3. The equivalent circuit of the variable coupling resonator shown on Fig. 1 .
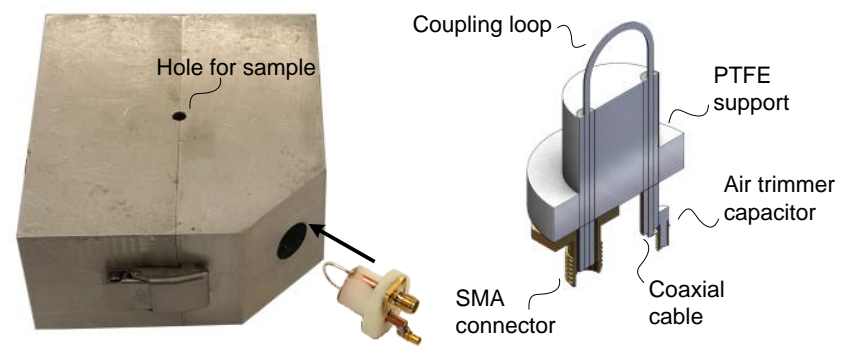

Fig. 4. Photograph and 3D drawing of the proposed variable coupling structure.

characteristic admittance and the electrical length of the coupling loop, respectively. The coupling can be controlled by changing the value of the variable capacitor.

An aluminum cylindrical cavity resonator was designed and fabricated with an internal radius of $46 \mathrm{~mm}$ and height of 40 $\mathrm{mm}$. The input was coupled from an inductive loop with diameter of $13 \mathrm{~mm}$, made from a silver-coated wire of $0.8 \mathrm{~mm}$ diameter. A liquid sample in a quartz capillary, which has an inner diameter of $1 \mathrm{~mm}$ and outer diameter of $1.2 \mathrm{~mm}$, was inserted through a hole along the cavity's axis. An air trimmer capacitor (47273, Johanson Manufacturing) with a tuning range of $0.6-4.5 \mathrm{pF}$ was used to vary the coupling. A photograph of the cylindrical resonator and the variable coupling circuit is shown in Fig. 4.

To find the value of the capacitance at a certain frequency, measurements when using open and short circuit loads (instead of the capacitor) are performed to find the $\mathrm{Z}$ parameters of the equivalent circuit as shown in Fig. 3.

\section{EXPERIMENTAL RESULTS AND DISCUSSION}

\section{A. Sensing mode resonator}

The sensing mode can be achieved by reducing the value of the coupling coefficient to increase the sensitivity (increase the quality factor). The magnitude of the $S_{11}$ was reduced to $10 \mathrm{~dB}$ by adjusting the value of the capacitor to $1.931 \mathrm{pF}$ at $2.499 \mathrm{GHz}$, as shown in Fig. 5. The corresponding $\left|\mathrm{S}_{11}\right|$ when using various liquids (water, methanol, ethanol, and chloroform) are shown in Fig. 5, the changes in the resonant frequency and quality factor being due to the effects of polarization and dielectric loss of the sample on the cavity resonance. The value of the complex permittivity of the sample can be extracted by using perturbation theory as in [10], [11]. 


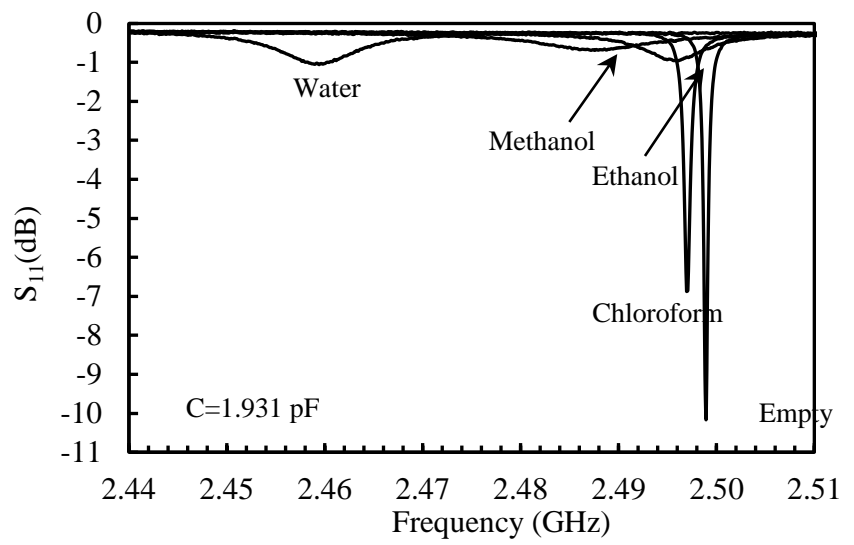

Fig. 5 Measured $\left|S_{11}\right|$ for several solvents at $25^{\circ} \mathrm{C}$ when the terminating capacitor is $1.931 \mathrm{pF}$ at $2.499 \mathrm{GHz}$ (for the sensing mode).

\section{B. Heating mode resonator}

As shown in Fig 6 (a), first, the capacitor is adjusted to give critical coupling $\left(\mathrm{S}_{11}=-40 \mathrm{~dB}\right.$ at $\left.C=1.878 \mathrm{pF}\right)$ in case of an empty capillary. When the sample is introduced into the cavity, chloroform (a weakly polar liquid) reduces the coupling to $-15 \mathrm{~dB}$. Strongly polar liquids such as water, methanol, and ethanol reduces the coupling down to $-1.5 \mathrm{~dB}$, since $Q_{o}$ Is much lower. Now, the amount of coupling can be adjusted by tuning the terminating capacitor as illustrated

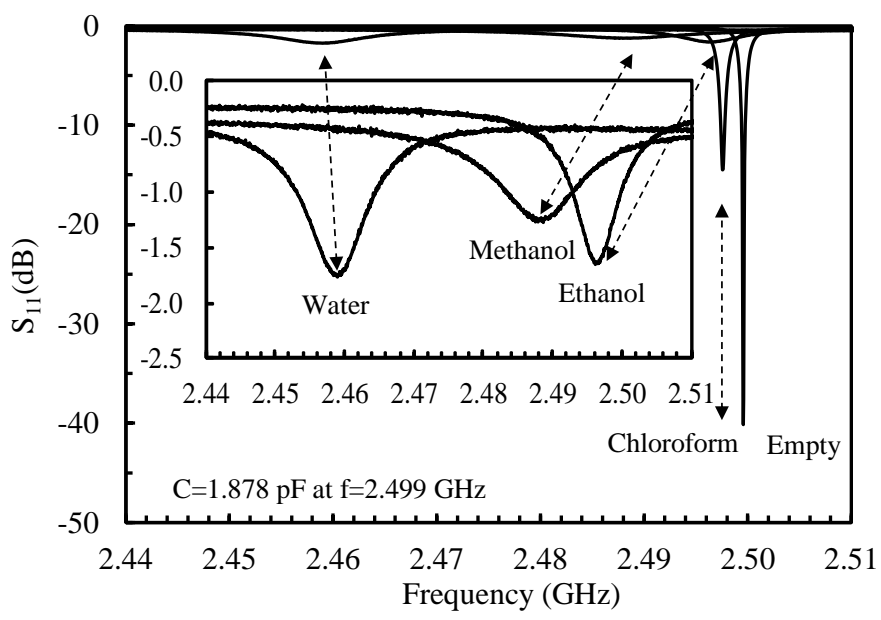

(a)

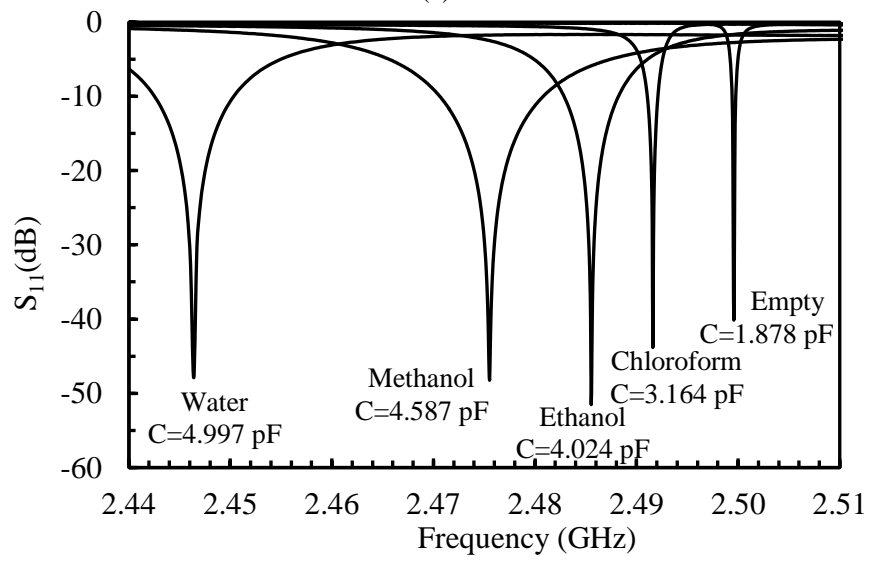

(b)

Fig. 6. Measured $\left|S_{11}\right|$ for several solvents at $25^{\circ} \mathrm{C}$. (a) before tuning, and (b) after tuning. in Fig. 6 (b). Critical coupling $\left(\left|S_{11}\right|\right.$ between at least $\left.-40 \mathrm{~dB}\right)$ is achieved for all solvents by setting different values of the capacitor. Critical coupling will make sure the power available from the source is delivered effectively to the sample, resulting in high efficiency and, therefore, fast heating. The values of the terminating capacitor are shown in Fig. 6 (b).

\section{CONCLUSIONS}

In this letter, a new technique for a variable coupling resonator is proposed. This technique not only shows the ability to maximize the coupling (reaching critical coupling) in the case of using the resonator for heating applications, but also provides the facility to use the resonator as sensor for liquid permittivity characterization. These two functions are required together in many medical, biological, and chemical applications. A cylindrical waveguide cavity at $\mathrm{TM}_{010}$ and resonant frequency of $2.45 \mathrm{GHz}$ was used to verify the variable coupling concept where the coupling loop was terminated by a variable capacitor to tune the coupling. Here, we used screwdriver tunable capacitor type to proof the validity of the new coupling technique. A varactor diode can be used instead to provide the facility to control the coupling electronically. The experimental results validated the concept of variable coupling technique.

\section{REFERENCES}

[1] M. Puentes, F. Bashir, M. Maasch, M. Schüßler, and R. Jakoby, "Planar microwave sensor for thermal ablation of organic tissue," in European Microwave Conference (EuMC), Nuremberg, 2013, pp. 479 - 482.

[2] M. Puentes, M. Schüßler, C. Damm and R. Jakoby, "Evolution of a microwave instrument for analysis and thermal ablation of organic tissue," in European Microwave Conference (EuMC), Rome, 2014, pp. $283-286$.

[3] M. S. Boybay, A. Jiao, T. Glawdel, and C. L. Ren, "Microwave sensing and heating of individual droplets in microfluidic devices," Lab Chip, vol. 13, no. 19, pp. 3840-3846, Oct. 2013.

[4] Y. Katsuta and Y. Nikawa, "Measurement of temperature dependent permittivity for thin material during microwave heating," in Asia-Pacific Microwave Conference (APMC), Bangkok, 2007, pp. 1-4.

[5] S. Jacobsen, P. R. Stauffer, and D. G. Neuman, "Dynamic measurement of dielectric properties of materials at high temperature during microwave heating in a dual mode cylindrical cavity," IEEE Trans.Microw. Theory Techn., vol. 63, no. 9, pp. 2905-2914, Sep. 2015

[6] I. Chaimov and S. R. Rogers, "Electromagnetic heating apparatus having decoupled excitations," in IEEE Int. Microw., Commun., Antennas, Electron. Syst. Conf., Tel Aviv, Israel, 2013, pp. 1-4.

[7] A. A. Abduljabar, H. Choi, D. A. Barrow, and A. Porch, "Adaptive coupling of resonators for efficient microwave heating of microfluidic systems," IEEE Trans.Microw. Theory Techn., vol. 63, no. 11, pp. 3681 - 3690, Nov. 2015.

[8] R. F. Harrington, Time-Harmonic Electromagnetic Fields. New York, USA: John Wiley and Sons, 2001

[9] M. Sánchez-Renedo, R. Gómez-García, J. I. Alonso, and C. BrisoRodríguez, "Tunable combline filter with continuous control of center frequency and bandwidth,l" IEEE Trans.Microw. Theory Techn., vol. 53, no. 1, pp. 191 - 199, Jan. 2005.

[10] M. Lin and M. N. Afsar, "A new cavity perturbation technique for accurate measurement of dielectric parameters," in IEEE MTT-S Int. Microw. Symp. Dig., 2006, pp. 1630-1633

[11] J. A. Cuenca, E. Thomas, S. Mandal, O. Williams, and A. Porch, "Investigating the broadband microwave absorption of nanodiamond impurities," IEEE Trans.Microw. Theory Techn., vol. 63, no. 12, pp. 4110 - 4118, Dec. 2015. 